Thromboembolien bei

Tumorpatienten

Prof. Dr. med. Christian Straka
Chefarzt der Abtlg. Hämatologie und Onkologie
Schön Klinik Starnberger See, Berg

47- Mit klinischem Gespür die Gefahr einschätzen

50 - Wie lässt sich eine venöse Thromboembolie verhindern?

\title{
Krebspatienten
}

\section{Welche Thromboseprophylaxe ist sinnvoll?}

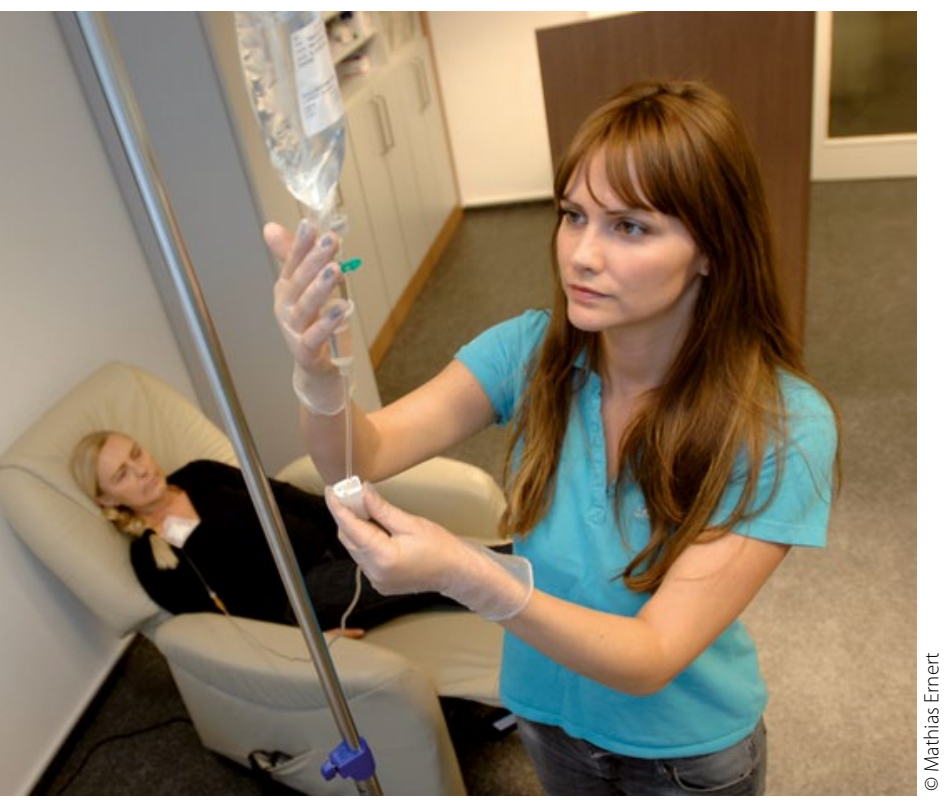

- Das Wissen um ein erhöhtes Thromboembolierisiko bei Tumorpatienten ist mittlerweile weit verbreitet. Welche praktischen Maßnahmen speziell der Thromboembolieprophylaxe sich sinnvollerweise daraus ergeben, ist aber nicht immer einfach zu beantworten. Die große Bedeutung der Frage wird klar, wenn man bedenkt, dass die Lungenembolie die zweithäufigste Todesursache von hospitalisierten Krebspatienten darstellt. Dazu kommt die nach einer venösen Thromboembolie (VTE) verminderte Lebensqualität. VTEs können auch zu Interferenzen mit einer systemischen Chemotherapie führen, die unter Umständen sogar eine kurative Behandlung gefährden können.

Inwieweit ist es also möglich, das Thromboserisiko bei Tumorpatienten zu quantifizieren, um die richtige Entscheidung über Notwendigkeit und genaue Art einer Thromboseprophylaxe treffen zu können? Einzelne Laborparameter, Laborkonstellationen oder auch neue Biomarker sind in der Regel zu vieldeutig und ohne klare
Schwellenwerte wenig brauchbar. Grundsätzliche Probleme von Scoresystemen sind die relative Willkür, mit der die dem Risikoscore zugrunde liegenden Parameter ausgewählt wurden, sowie die häufig fehlende oder nur fragmentarisch erfolgte Validierung. Klare Anhaltspunkte bieten dann doch wieder einzelne, definierte Hochrisikoparameter wie z. B. eine bereits vorangegangene tiefe Beinvenenthrombose.

Letztendlich bleibt die klinische Erfahrung des behandelnden Arztes und seine Gesamteinschätzung des Risikos entscheidend. Der behandelnde Arzt ist aber auch mit der anhaltenden Unsicherheit im Einzelfall konfrontiert, zumal sich das Risikoprofil im Verlauf durch neu auftretende oder wegfallende Faktoren wieder verändern kann. Konsens herrscht darüber, dass hospitalisierte Krebspatienten ein Hochrisikokollektiv für Thromboembolien darstellen und deswegen eine konsequente prophylaktische Antikoagulation indiziert ist.

Bestimmte Tumorerkrankungen sind mit einem erhöhten Thromboembolierisiko verbunden, so das Pankreaskarzinom, das Magenkarzinom und auch myeloproliferative Erkrankungen mit erhöhter Blutzellzahl. Auch neuere Therapieformen wie die Inhibitoren der Angiogenese sind hier zu nennen. Beim multiplen Myelom führt die Anwendung von Thalidomid oder Lenalidomid zu einem stärker erhöhten Thromboserisiko, sodass eine Thromboseprophylaxe auch im ambulanten Sektor über einen längeren Zeitraum erfolgen muss. Chirurgische Eingriffe sind mit einem additiven Thromboserisiko, aber gleichzeitig mit einem Blutungsrisiko verbunden.

Nationale und internationale Empfehlungen zur Durchführung einer Thromboseprophylaxe sind nicht immer einheitlich. Die niedermolekularen Heparine bieten eine Reihe von Vorteilen, Vitamin KAntagonisten sind dagegen bei Tumorpatienten eher problematisch, da plötzlich invasive Eingriffe notwendig werden oder auch die Thrombozytenwerte unter Chemotherapie stärker abfallen können. Die als Weiterentwicklung zu betrachtenden neuen oralen Faktor Xa- und Faktor Ila-Antagonisten sind vielversprechende Entwicklungen, deren Nutzen aber noch nicht abschließend beurteilt werden kann. 